\title{
Evolving Role of Magseed in Wireless Localization of Breast Lesions: Systematic Review and Pooled Analysis of 1,559 Procedures
}

\author{
RITIKA GERA $^{1}$, SALIM TAYEH $^{1}$, SARA AL-REEFY $^{2}$ and KEFAH MOKBEL ${ }^{1}$ \\ ${ }^{1}$ London Breast Institute, The Princess Grace Hospital, London, U.K.; \\ ${ }^{2}$ London Breast Care Centre, Manama, Bahrain
}

\begin{abstract}
Background/Aim: Wire-guided localisation (WGL) remains the most widely used technique for the localisation of non-palpable breast lesions; however, recent technological advances have resulted in non-wire, non-radioactive alternatives, such as magnetic seeds (Magseeds). The aim of this pooled analysis was to determine whether Magseeds are an effective tool for localising non-palpable breast lesions. Materials and Methods: Various databases were searched for publications which reported data on the localisation and placement rates of Magseed. Data on re-excision rates under use of Magseed and WGL were also collected. Results: Sixteen studies, spanning the insertion of 1,559 Magseeds, were analysed. The pooled analysis showed a successful placement rate of $94.42 \%$ and a successful localisation rate of $99.86 \%$. Four studies were analysed in a separate pooled analysis and showed no statistically significant difference between reexcision rates using Magseeds and WGL. Conclusion: The use of Magseeds is an effective, non-inferior alternative to WGL that overcomes many of the limitations of the latter.
\end{abstract}

The pre-operative localisation of non-palpable breast cancer traditionally employs wire-guided localisation (WGL) (1). However, several limitations are associated with WGL. The localisation wire can cause manifold complications, such as diathermy burns, pericardial injury and wire dislocation/ transection $(2,3)$. Furthermore, wire migration and surgical difficulties in accurately assessing the position of the wire tip intraoperatively can pose significant difficulty for the

This article is freely accessible online.

Correspondence to: Professor Kefah Mokbel, 42-52 Nottingham Pl, Marylebone, London W1U 5NY, U.K. Tel: +44 2079082040, Fax: +442079082275, e-mail: kefahmokbel@hotmail.com

Key Words: Magseed, pooled analysis, wireless, localisation, review. operating surgeon (4). WGL is performed on the day of surgery, and inter-departmental coordination with radiology for localisation in addition to everyday scheduling difficulties may lead to a prolonged surgical waiting time. An underreported limitation of the flexible wire used in WGL is that it increases the risk of needle-stick injury for the surgeon and the pathologist. Furthermore, it protrudes from the breast and is uncomfortable for the patient. The prospect of an additional procedure on the day of major breast surgery can also be very stressful for patients in whom anxiety level is already high (2).

Widespread use of screening methods has led to an increased incidence of non-palpable breast cancer. It is therefore imperative that localisation methods with high patient, radiologist and surgical satisfaction rates are developed.

Radioactive seed localisation (RSL) is a feasible alternative to WGL. RSL can occur up to 5 days prior to the surgery and the seeds can be accurately detected with a hand-held gamma probe (5). Furthermore, recent evidence suggests that RSL would be more cost-effective than WGL (6) and would improve the oncological outcomes of imageguided surgery (7). However, handling of radioactive material requires special licensing and is associated with strict regulatory requirements. Hence, the optimal solution would be a non-wire non-radioactive localisation method which contains no energy source. This has led to the emergence of Savi Scout (Cianna Medical Inc., Aliso Viejo, CA, USA) (8) - a Food and Drug Administration-approved and CounciI of Europe-marked reflector that uses micro impulse radar; magnetic seeds (Magseed; Endomagnetics Inc., Cambridge, UK) (9); and radiofrequency identification (RFID) tags (10) (LOCalizer; Hologic, Santa Carla CA, USA). In addition to overcoming many of the limitations of WGL and allowing flexible scheduling by decoupling surgery and radiology, these wireless options allow marker deployment at the time of biopsy and can be very useful in patients undergoing neoadjuvant systemic therapy. The limitations of these wireless options compared with WGL 
include the inability to adjust the position of the marker once deployed and lack of magnetic resonance imaging (MRI)compatible introducer needles.

The Magseed technique is the focus of this article. A Magseed is an inducible non-wire non-radioactive paramagnetic seed which is made of surgical grade stainless steel (Figure 1). The seed is introduced through a sterile needle using ultrasound or mammography guidance. The detector probe magnetizes the iron within the seed and transiently creates a magnetic field. The probe then detects the magnetization produced by the seed to provide real-time localization. Although detection is reliable within $4 \mathrm{~cm}$ depth from the skin surface, the manufacturer reported detection at up to $12 \mathrm{~cm}$ through palpation in the supine position. The seed can be placed inside the breast lesion at any time prior to surgery (Figure 2). The Sentimag probe induces and detects the magnetic field of a Magseed with audio signals and numerical values that correlate with distance of the seed from the probe, thus allowing continuous reorientation (11) (Figure 3). However, during the use of the probe, metal instruments containing iron need to be moved away from the tip of the detection probe and this represents a disadvantage of this technique. We recently demonstrated that use of Magseed, is an effective alternative to WGL with a high acceptance rate among patients, radiologists and surgeons (9). The use of Magseed technology for lesion localisation gained US Food and Drug Administration approval in 2016 and was CounciI of Europe marked in 2017. Moreover, it was recently approved by regulators for long-term deployment at any time before surgery and for deployment within axillary lymph nodes.

The aim of this systematic review and pooled analysis was to assess the effectiveness of Magseeds for lesion localisation in non-palpable breast cancer and investigate whether their use was a non-inferior alternative to WGL. This analysis focused on three variables: Successful placement of Magseeds, successful identification and retrieval of Magseeds, and lesion margin positivity/re-excision rates.

\section{Materials and Methods}

Data sources and searches. A complete search of the PubMed, Ovid, Google Scholar, and Cochrane databases was undertaken to identify relevant publications. The search terms differed depending on the database. The PubMed search was carried out on 11 th August 2019 with the search criteria 'magnetic seed AND breast'. The Ovid search used the AMED, Embase, and Ovid MEDLINE(R) and Epub Ahead of Print, In-Process and Other Non-Indexed Citations, Daily and Versions[R] databases on $1^{\text {st }}$ September 2019 to isolate texts which matched the search term 'magnetic seeds'. The Google Scholar search, which was performed on $18^{\text {th }}$ August 2019, used the search term 'Magseed localisation' to find relevant publications. Finally, the Cochrane search used the term 'magnetic seed breast cancer' on $16^{\text {th }}$ August 2019 to identify appropriate texts.
Inclusion and exclusion criteria. Prospective clinical trials and retrospective studies were included. In the abstract, the publication needed to have summarised the findings of their centre when exploring the localisation of non-palpable breast lesions using Magseed technology. In the full-text, the following raw data had to be included: Total number of patients undergoing Magseed localisation, successful placement/localisation of the Magseed, and successful identification/retrieval of the Magseed. Data regarding re-excision rates were also extracted and analysed when available. When an article detailed margin positivity, we assumed this indicated re-excision, hence margin positivity rates were also used for calculating re-excision rates. Previously published systematic reviews were thoroughly examined for any relevant publications which might be added to our search results. Abstracts and full texts were included in this analysis, and abstracts from conferences were also sent to us by colleagues.

Abstracts were excluded for the overall pooled analysis when they did not clearly examine the use of Magseeds to localise nonpalpable breast lesions. Abstract/full-text publications were excluded from the overall pooled analysis when data regarding the successful placement/localisation or identification/retrieval of the Magseeds were unavailable. However, when publications directly compared WGL with Magseeds, they were included in a separate pooled analysis. Where possible, we attempted to contact the lead author to request missing data.

Data management. Data were extracted independently by the Authors and combined to calculate the overall rates of successful placement/localisation and successful identification/retrieval using data sets from included studies. Where no extra data were provided, it was assumed that the number of patients was equal to the number of Magseeds placed. Data sets from each included study were combined to calculate mean values for the rates of successful placement, localisation and re-excision. The localisation rate was calculated using only Magseeds which had successfully been placed.

\section{Results}

Literature search results and characteristics of the included studies. A total of 179 studies were initially assessed (58 from PubMed, 49 from Ovid, five from the Cochrane Library, and 67 from Google Scholar). A total of 161 were immediately excluded when the abstracts were screened. Many were reviews; hence they were thoroughly examined for any pertinent randomized controlled or retrospective trial which had not been found in our initial search and could be included in the pooled analysis. Two abstracts and one full text were also added to our analysis through an external source.

We then proceeded to screen the full texts, when available, for the 21 abstracts which initially met the criteria for analysing Magseed localisation efficacy (9, 11-30). Five studies were excluded from the localisation/identification rate calculation because these data was not provided or were unclear $(12,15,21)$ or because the total number of Magseeds used was unclear $(13,22)$. Hence, 16 studies were used to calculate the rates of successful localisation and placement 


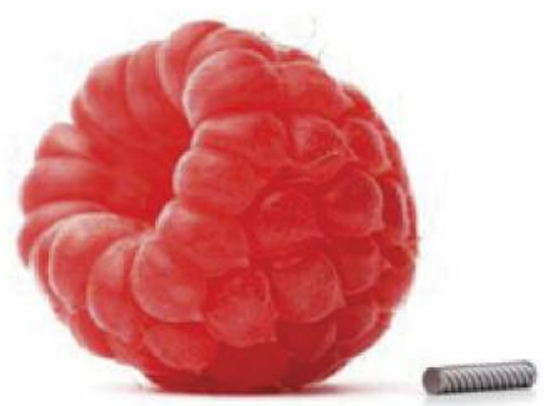

Figure 1. An illustration of the size and scale of the Magseed relative to a raspberry. The Magseed measures $5 \mathrm{~mm}$ in size.

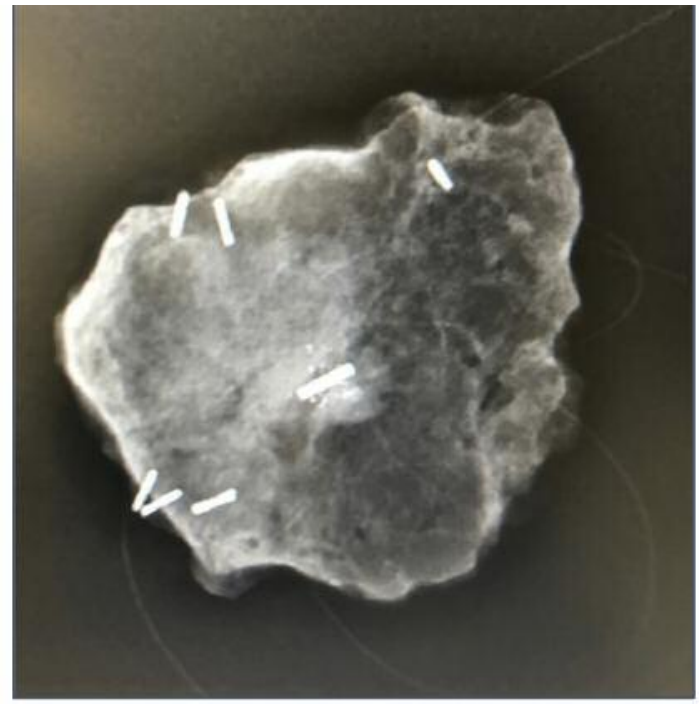

Figure 2. Example of a lesion that was excised after Magseed localisation. Specimen mammography demonstrating the Magseed in the centre of screening-detected breast cancer. The peripheral metal clips were deployed in order to orientate the specimen. The final margins were tumour-free in this case.

in the pooled analysis $(9,11,14,16-20,23-30)$. Twelve studies were used to calculate the overall re-excision rate $(9$, $11,14,18,20,23-30)$.

Four studies were included in a smaller pooled analysis which selectively examined the use of Magseeds in direct comparison to WGL $(12,13,22,30)$. This analysis included some studies excluded from the larger pooled analysis because relevant data were found directly comparing the two methods.

These results are summarised in a PRISMA flow chart (Figure 4).

Results of the pooled analysis. Across the 16 studies included in the overall pooled analysis, 1,559 Magseeds were inserted, from which 1,470 Magseeds were successfully deployed and

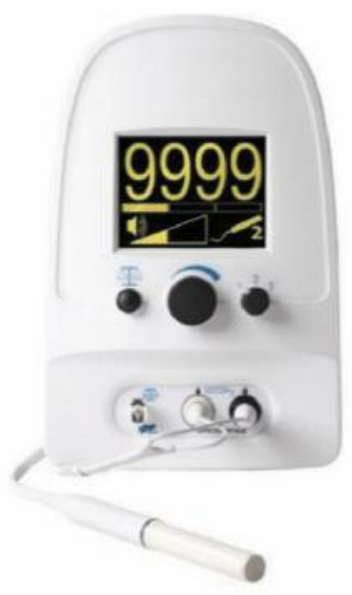

Figure 3. Example of a Sentimag probe.

1,468 Magseeds were successfully localised. Hence, the successful placement rate was $94.42 \%$ and the successful localisation rate was $99.86 \%$. In publications which included data on re-excision rates, 1,483 Magseeds were inserted and 166 re-excisions were recorded. Hence, the re-excision rate was $11.19 \%$.

Finally, across the four studies directly comparing WGL to Magseeds, 319 Magseeds were inserted and 507 WGLs were performed. In these studies, 59 Magseeds had to be reexcised compared to 82 WGLs. Hence, the Magseed reexcision rate was $18.50 \%$ and the WGL re-excision rate was $16.17 \%$. A chi-square test determined that this difference was not statistically significant (chi-square statistic with Yates' correction $=0.5906, p=0.442192$ ).

\section{Discussion}

The high successful localisation and retrieval rate of $99.86 \%$ and relatively low re-excision rate of $11.25 \%$ support the effective utilization of Magseed as a valid safe alternative to WGL while overcoming many of the limitations of the latter. The lack of adequately powered randomised clinical trials prompted this pooled analysis, which included data on 1,559 Magseed cases. The results reflected favourably on the use of Magseed technology for the effective localisation of nonpalpable breast lesions.

We initially aimed to include data from publications which defined successful localisation/placement as within $1 \mathrm{~cm}$ of the lesion. However, most publications did not provide clarification on their definition of successful localisation/placement. Hence, a significant limitation of our pooled analysis is that we included data sets with heterogeneous measurements of technically successful deployments and localisations. Furthermore, surgeons and radiologists across different institutions have variable levels 


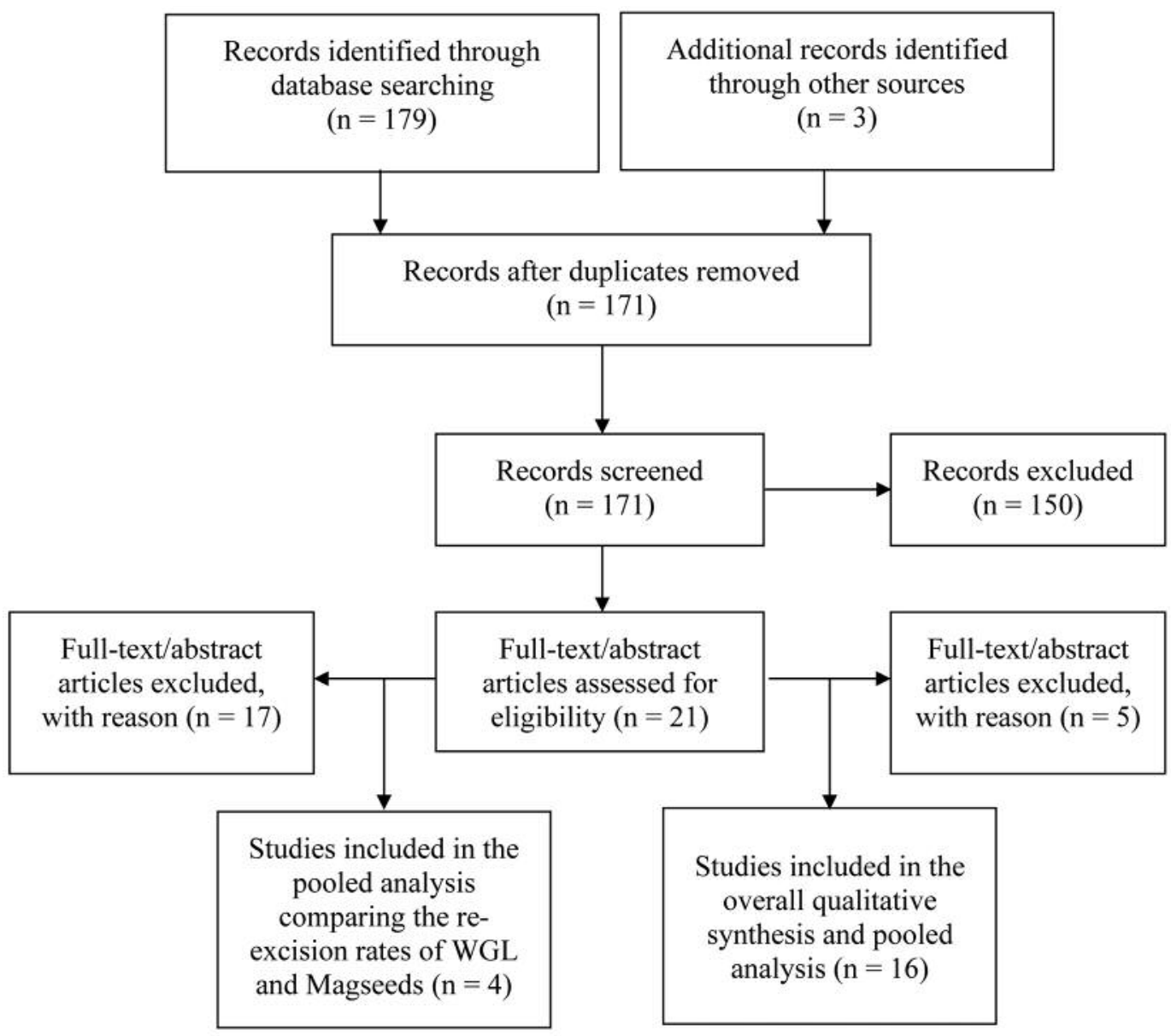

Figure 4. A PRISMA flowchart summarising the results of data collection. WGL: Wire-guided localisation.

of expertise and participate in the care of heterogeneous patient groups, hence many factors may have confounded the data sets used for this pooled analysis. Lack of standardization across studies precluded the performance of a pooled analysis regarding other important variables such the migration rate, depth of lesions, or specimen weight. However, individual studies reported minimal migration of magnetic seeds (9) and no difference in specimen size compared with WGL (28).

The re-excision rate of $11.25 \%$ found in our analysis seems to be lower than that reported in large series for WGL, where the margin positivity rate ranged from $16.4 \%$ to $20 \%$ (31-33). This difference might be partially attributed to the fact that centres reporting Magseed experience are more likely to be academic with a high volume of cases and have greater radiological and surgical expertise than others. However, we found no significant difference in the reexcision rates between Magseed and WGL in the separate pooled analysis of four suitable studies $(12,13,22,30)$.
Since the use of Magseeds is a new technique, it is feasible that the learning curve may have adversely affected the reexcision rates in these initial evaluation studies.

Compared to WGL, the use of Magseeds has many discernible benefits. Seed deployment in advance of surgery increases scheduling flexibility and possibly reduces overall cost due to more efficient theatre and radiology suite utilisation. Although Magseed use is more expensive than WGL (30), the cost-effectiveness demonstrated with use of radioactive seeds is likely to translate to that with Magseeds (6) and further research is required to confirm this. Furthermore, a feasibility study comparing magnetic markers to radioactive seeds reported positive findings in support of the technology as a viable non-radioactive alternative to current techniques (19).

Although Magseeds are detectable in all breast sizes, seeds which were placed closer to the skin surface were more likely to be detected, and a significant correlation was found between breast weight and recorded probe count. This 
indicates that the use of Magseeds may have some limitations in patients with deep $(>6 \mathrm{~cm})$ non-palpable lesions (16). Furthermore, stainless steel surgical instruments can interfere with detection of magnetic seeds, and using non-magnetisable (titanium, polymer) alternatives may increase operating costs and time (34).

Another non-wire non-radioactive localisation alternative is that of RFID tags. A ferrite rod covered with copper and wrapped by a microprocessor and glass casing (34), the RFID tag can also be deployed prior to breast-conserving surgery and is safe and effective for non-palpable lesion localisation (35). However, although re-excision rates between RFID tags and WGL were generally similar, there was a significantly higher re-excision rate and greater specimen size for bracketed ductal carcinoma in situ lesions when using an RFID tag (36). The RFID tags used with LOCalizer are larger than Magseeds (12 vs. $5 \mathrm{~mm}$ ) and are currently deployed through a wider introducer needle (12-gauge vs. 8-gauge) (10). However, the detection equipment is lighter and more compact than Sentimag and measures the distance between the probe and marker (with an accuracy of $\pm 7 \mathrm{~mm}$ according to the premarket notification for LOCalizer) during real time navigation.

A significant limitation impeding the use of both RFID tags and Magseeds as localisation techniques is that both can result in signal void artefacts (2 and $4 \mathrm{~cm}$, respectively) during follow-up MRI scans (37); depending on the MRI sequence, the Magseed can create a bloom artefact which measures up to $4 \mathrm{~cm}$. This may hamper the detection of residual disease after neoadjuvant systemic therapy if MRI is required for monitoring response to treatment. Nevertheless, contrast-enhanced mammography is an effective alternative imaging modality which may be considered if signal void artefacts pose a significant issue (38).

The Savi Scout system is another non-wire nonradioactive alternative. A reflector which uses a microimpulse infrared radar for lesion localisation (39), the Savi Scout system does not generate MRI phantoms and therefore can be deployed at the time of biopsy in patients undergoing neoadjuvant systemic therapy that requires MRI monitoring (40). However, the Savi Scout system may fail to generate an audible signal when used for the localisation of lesions at an excessive depth within the breast or associated with a haematoma $(40,41)$. In comparison with Magseeds, the Savi Scout utilizes a 16-gauge introducer needle (versus 18-gauge for Magseeds) and the current reflector is larger in size $(12 \times 1.6 \mathrm{~mm}$ versus $5 \times 0.9 \mathrm{~mm})$. Therefore, Magseeds might be preferred for small superficial lesions close to the skin. Furthermore, the Magseed introducer needle is appropriately calibrated for immediate deployment during stereotactic vacuum-assisted biopsy, whereas the current introducer needle for Savi Scout was not designed for this purpose.
Two publications focused specifically on clinician satisfaction and patient anxiety outcomes for Magseeds compared to WGL $(13,21)$. A prospective interim analysis evaluating clinical outcome and clinician satisfaction with Magseeds compared to WGL reported statistically significant and favourable outcomes for Magseeds: $84.9 \%$ of clinicians found Magseeds to be easy/very easy to localise as opposed to $64.5 \%$ for WGL. Furthermore, transcutaneous localisation was easy/very easy for $80.4 \%$ of the cases using Magseeds compared to $59.2 \%$ of the wire-guided cases, and intraoperative localisation was easy/very easy at $80 \%$ compared with $64.7 \%$ of cases, respectively. Our more recently reported observations (9) are consistent with these findings. Interestingly, although the study radiologists and surgeons overwhelmingly preferred the use of Magseeds as their localisation technique of choice, there was no difference in patient satisfaction between the two (13).

However, a separate analysis of patient anxiety related to Magseeds and WGL used standardised state trait anxiety questionnaires to investigate any patterns of the association between localisation method and subsequent patient anxiety (21). Although significance values were not calculated, scalebased average scores were 36 for Magseeds and 41 for WGL, suggesting lower pre-operative anxiety when patients were faced with the Magseed as their localisation technique. This study was not included in the pooled analysis because no data were provided regarding the rates of re-excision.

\section{Future Directions}

It would be interesting to further investigate the localisation and placement rate of Magseeds when combined with superparamagnetic iron oxide (SPIO) particles for sentinel lymph node detection. In tumours located deep in the breast, SPIO particles seem to amplify the transcutaneous magnetic signal (17), which might aid the use of Magseeds in patients with larger breasts or deeper lesions (16). However, SPIO has been linked to artefact generation in MRIs, hence its use in combination with the Magseed system should be investigated with caution (42). As MRI-void signals pose a significant problem for Magseed use, there is a need for their reduction and for the development of MRI-compatible introducer needles. Furthermore, according to the manufacturer, the next generation of the Magseed-Sentimag device utilizes a lighter and slimmer probe that also measures the distance of the magnetic seed from the probe, thus facilitating a more accurate excision. Second-generation magnetic seeds are expected to generate smaller void signals on MRI.

Future studies should include a quantitative cost-effective analysis to determine whether the use of Magseeds is fiscally superior to WGL in the long term. Improvement of the aesthetic outcome is an important consideration for the patient and should also be further investigated. 


\section{Conclusion}

There is a growing body of evidence that Magseeds are a non-inferior, reliable and effective radiation-free localisation alternative to WGL. They are widely accepted by surgeons, radiologists and patients, and facilitate increased operating efficiency due to the decoupling of radiology and surgery schedules. There is currently no evidence that the use of Magseeds is associated with lower re-excision rates and specimen size compared with WGL.

\section{Conflicts of Interest}

This work carried no conflicts of interest.

\section{Authors' Contributions}

Gera R carried out the data search. Gera R and Mokbel K wrote the article. Gera R, Mokbel K, Al-Reefy S and Tayeh S reviewed the article.

\section{References}

1 DeSantis CE, Fedewa SA, Goding Sauer A, Kramer JL, Smith RA and Jemal A: Breast cancer statistics, 2015: Convergence of incidence rates between black and white women. CA Cancer J Clin 66(1): 31-42, 2016. PMID: 26513636. DOI: 10.3322/caac. 21320

2 Dua SM, Gray RJ and Keshtgar M: Strategies for localisation of impalpable breast lesions. The Breast 20(3): 246-253, 2011. PMID: 21285000. DOI: 10.1016/j.breast.2011.01.007

3 Seifi A, Axelrod H, Nascimento T, Salam Z, Karimi S, Avestimehr S and Ohebsion J: Migration of guidewire after surgical breast biopsy: An unusual case report. Cardiovasc Intervent Radiol 32(5): 1087-1090, 2009. PMID: 19506948. DOI: $10.1007 /$ s00270-009-9620-9

4 Rahusen FD, Bremers AJA, Fabry HFJ, Taets van Amerongen AHM, Boom RPA and Meijer S: Ultrasound-guided lumpectomy of nonpalpable breast cancer versus wire-guided resection: A randomized clinical trial. Ann Surg Oncol 9(10): 994-998, 2002. PMID: 12464592 . DOI: $10.1007 / b f 02574518$

5 Langhans L, Tvedskov TF, Klausen TL, Jensen M-B, Talman ML, Vejborg I, Benian C, Roslind A, Hermansen J, Oturai PS, Bentzon N and Kroman N: Radioactive seed localization or wire-guided localization of nonpalpable invasive and in situ breast cancer. Ann Surg 266(1): 29-35, 2017. PMID: 28257326. DOI: $10.1097 /$ SLA.0000000000002101

6 Zhang Y, Seely J, Cordeiro E, Hefler J, Thavorn K, Mahajan M, Domina S, Aro K, Ibrahim AM, Arnaout A, Gravel D and Nessim C: Radioactive seed localization versus wire-guided localization for nonpalpable breast cancer: A cost and operating room efficiency analysis. Ann Surg Oncol 24(12): 3567-3573, 2014. PMID: 28913761. DOI: 10.1245/s10434-017-6084-z

7 Pieri A, Milligan R, Critchley A, O’Donoghue JM, Sibal N, Peace R, Petrides G, Howitt R, Nicholson S and Cain H: The introduction of radioactive seed localisation improves the oncological outcome of image-guided breast-conservation surgery. The Breast 36: 49-53, 2017. PMID: 28945991. DOI: 10.1016/j.breast.2017.09.004
8 SAVI SCOUT ${ }^{\circledR}$ Highlighted as a Cutting Edge Technology for Breast Cancer Patients in 2018 - Cianna Medical, powered by Merit Medical [Internet]. Available from: https://www.cianna medical.com/cianna_news_releases/savi-scout-highlighted-as-acutting-edge-technology-for-breast-cancer-patients-in-2018

9 Tayeh S, Gera R, Perry N, Michell M, Malhotra A and Mokbel K: The use of magnetic seeds and radiofrequency identifier tags in breast surgery for non-palpable lesions. Anticancer Res 40(1): 315321, 2020. PMID: 31892582. DOI: 10.21873/anticanres.13955

10 Wazir U, Tayeh S, Perry N, Michell M, Malhotra A and Mokbel K. Wireless breast localization using radio-frequency identification tags: The first reported European experience in breast cancer. In Vivo 34(1): 233-238, 2020. PMID: 31882483. DOI: 10.21873 /invivo. 11765

11 Price ER, Khoury AL, Esserman LJ, Joe BN and Alvarado MD: Initial clinical experience with an inducible magnetic seed system for preoperative breast lesion localization. Am J Roentgenol 210(4): 913-917, 2018. PMID: 29446680. DOI: 10.2214/AJR.17.18345

12 Crane J, Photi E and Down S: A DGH's experience using Magseed as a localisation tool for impalpable breast lesions. Eur J Surg Oncol 45(5): 923-924, 2019. DOI: 10.1016/j.ejso.2019.01.172

13 Micha A, Sinnett V, Wilson R, Adams E, Patrick E, Hector L, Chamberlain C, Edmonds R, Bishop B, Gui G, MacNeill F, Roche N, Tasoulis M, Allum W, Barry P, Rusby J and Krupa K: Interim analysis of an evaluation of clinical outcome and patient and clinician satisfaction with magnetic seeds compared with guide wires for localisation of impalpable breast lesions for surgery. Eur J Surg Oncol 45(5): 884, 2019. DOI: 10.1016/j.ejso.2019.01.216

14 Reid J, Tovey S, Campbell EJ, Pareek S, Kelly J, Law P, Coldeway J, Mumba E and Elgammal S: The MagSeed ${ }^{\circledR}$ experience: One year on. Eur J Surg Oncol 45(5): 884, 2019. DOI: 10.1016/j.ejso.2019.01.215

15 Sirianni C, Cochrane R, Gash A, Gate T, Gandakasuma B, Khattak I, Powell M, Abou-Samra W and Pennick M: The use of magnetic seeds for lesion localisation in breast surgery - The North Wales experience. Eur J Surg Oncol 45(5): 925, 2019. DOI: 10.1016/j.ejso.2019.01.177

16 Harvey JR, Lim Y, Murphy J, Howe M, Morris J, Goyal A and Maxwell AJ: Safety and feasibility of breast lesion localization using magnetic seeds (Magseed): A multi-centre, open-label cohort study. Breast Cancer Res Treat 169(3): 531-536, 2018. PMID: 29453521. DOI: 10.1007/s10549-018-4709-y

17 Hersi A-F, Eriksson S, Ramos J, Abdsaleh S, Wärnberg F and Karakatsanis A: A combined, totally magnetic technique with a magnetic marker for non-palpable tumour localization and superparamagnetic iron oxide nanoparticles for sentinel lymph node detection in breast cancer surgery. Eur J Surg Oncol 45(4): 544-549, 2019. PMID: 30401506. DOI: 10.1016/j.ejso.2018.10.064

18 Lamb LR, Bahl M, Specht MC, D'Alessandro HA and Lehman $\mathrm{CD}$ : Evaluation of a nonradioactive magnetic marker wireless localization program. Am J Roentgenol 211(4): 940-945, 2018. PMID: 30085842. DOI: 10.2214/AJR.18.19637

19 Schermers B, van der Hage JA, Loo CE, Vrancken Peeters MTFD, Winter-Warnars HAO, van Duijnhoven F, Ten Haken B, Muller SH and Ruers TJM: Feasibility of magnetic marker localisation for non-palpable breast cancer. The Breast 33: 5056, 2017. PMID: 28282587. DOI: 10.1016/j.breast.2017.03.003

20 Thekkinkattil D, Kaushik M, Hoosein MM, Al-Attar M, Pilgrim S, Gvaramadze A, Hyklova L and Jibril A: A prospective, single- 
arm, multicentre clinical evaluation of a new localisation technique using non-radioactive Magseeds for surgery of clinically occult breast lesions. Clin Radiol 74(12): 974.e7-974.e11, 2019. PMID: 31563293. DOI: 10.1016/j.crad.2019.08.018

21 Small S, McAdam A and Mathers H: Analysis of patient anxiety related to magseed and guide-wire localisation techniques. Eur J Surg Oncol 45(5): 925, 2019. DOI:10.1016/j.ejso.2019.01.176

22 Spivey TL, Lamb LR, O.V. S, Lehman CD, Hughes KS, Gadd MA, Smith BL and Specht MC: Magnetic seed localization as an alternative to wire localization for non-palpable breast lesions. Soc Surg Oncol 71st Annual Cancer Symposium p. S9192, 2018.

23 Miller M, Li P, Freyvogel M, Greenwalt I, Rock L, Shenk R, Peterson E, Teresczuk M, Thompson C and Dietz J: Hospital system rollout and initial experience with stainless steel magnetised seeds for breast and lymph node localisation. Breast Surgeons Meeting 581631, 2019.

24 Elgammal S, Campbell E, Tovey S, Henderson S, Kelly J, Coldeway $\mathrm{J}$ and Reid $\mathrm{J}$ : Introducing magnetic seed localisation for impalpable breast cancer; A pioneering Scottish experience. Eur J Surg Oncol 45(2): e108, 2019. DOI: 10.1016/j.ejso.2018.10.374

25 Faridová A, Rauš K and Šírová R: New localisation technique for early stages of breast cancer using magnetic seeds. The Breast 44: S105, 2019. DOI: 10.1016/S0960-9776(19)30357-1

26 Lake B, Wilson M, Williams S, Metelko M, Thomas G, Lewis $\mathrm{S}$, Norwood L and Lewis T: Magseed for localisation of impalpable breast cancers is associated with high patient satisfaction and lower re-excision rates. Eur J Surg Oncol 44(11): 1839, 2018. DOI: 10.1016/j.ejso.2018.10.030

27 Singh P, Scoggins M, Sahin A, Hwang R, Kuerer H, Caudle A, Mittendorf E, Thompson A, Bedrosian I, Teshome M, SeSynder $\mathrm{S}$, Meric-Bernstam F and Hunt K: Effectiveness and safety of Magseed-localization for excision of breast lesions: A prospective clinical trial. ACS Quality and Safety Conference, 2019.

28 Smeets A, Nevelsteen I, Keupers M, Neven P, Prevos R and Van Ongeval C: Magnetic seed localization for nonpalpable breast lesions: First experiences. Eur J Cancer 92: S73, 2018. DOI: 10.1016/S0959-8049(18)30444-1

29 Taruno K, Nakamura S, Shigenaga R, Ide Y, Kuwayama T, Akashi S, Kurita T, Takei H, Sekino M and Kusakabe M: The new approach for surgery using magnetic marker system and magnetic probe for localization of non-palpable lesions of breast in Japan. The Breast 44: S111-1112, 2019. DOI: 10.1016/S09609776(19)30378-9

30 Zacharioudakis K, Down S, Bholah Z, Lee S, Khan T, Maxwell AJ, Howe M and Harvey J: Is the future magnetic? Magseed localisation for non palpable breast cancer. A multi-centre non randomised control study. Eur J Surg Oncol 45(11): 2016-2021, 2019. PMID: 31288944. DOI: 10.1016/j.ejso.2019.06.035

31 Langhans L, Jensen MB, Talman MLM, Vejborg I, Kroman N and Tvedskov TF: Reoperation rates in ductal carcinoma in situ $v s$. invasive breast cancer after wire-guided breast-conserving surgery. JAMA Surg 152(4): 378-384, 2017. PMID: 28002557. DOI: $10.1001 /$ jamasurg.2016.4751

32 Haloua MH, Volders JH, Krekel NMA, Barbé E, Sietses C, Jóźwiak K, Meijer S and van den Tol MP: A nationwide pathology study on surgical margins and excision volumes after breastconserving surgery: There is still much to be gained. Breast 25: 14-21, 2016. PMID: 26801411. DOI: 10.1016/j.breast.2015.11.003
33 Laws A, Brar MS, Bouchard-Fortier A, Leong B and Quan ML: Intraoperative margin assessment in wire-localized breastconserving surgery for invasive cancer: A Population-level comparison of techniques. Ann Surg Oncol 23(10): 3290-3296, 2016. PMID: 27406094. DOI: 10.1245/s10434-016-5401-2

34 Friedewald S: Breast imaging, an issue of radiologic clinics of North America, Volume 55-3, 1st Edition. Elsevier, 2017.

35 Dauphine C, Reicher JJ, Reicher MA, Gondusky C, Khalkhali I and Kim M: A prospective clinical study to evaluate the safety and performance of wireless localization of nonpalpable breast lesions using radiofrequency identification technology. Am J Roentgenol 204(6): W720-723, 2015. PMID: 26001262. DOI: 10.2214/AJR.14.13201

36 McGugin C, Spivey T, Coopey S, Smith B, Kelly B, Gadd M, Hughes K, Dontchos B and Specht M: Radiofrequency identification tag localization is comparable to wire localization for non-palpable breast lesions. Breast Cancer Res Treat 177(3): 735-739, 2019. PMID: 31302856. DOI: 10.1007/s10549-01905355-0

37 Hayes MK: Update on preoperative breast localization. Radiol Clin North Am 55(3): 591-603, 2017. PMID: 28411682. DOI: 10.1016/j.rcl.2016.12.012

38 Barra FR, Sobrinho AB, Barra RR, Magalhães MT, Aguiar LR, Albuquerque GFL de, Costa RP, Farage L and Pratesi R: Contrast-enhanced mammography (CEM) for detecting residual disease after neoadjuvant chemotherapy: a comparison with breast magnetic resonance imaging (MRI). Biomed Res Int 2018: 1-9, 2018. DOI: 10.1155/2018/8531916

39 Mango V, Ha R, Gomberawalla A, Wynn R and Feldman S: Evaluation of the SAVI SCOUT surgical guidance system for localization and excision of nonpalpable breast lesions: A feasibility study. Am J Roentgenol 207(4): W69-72, 2016. PMID: 27304083. DOI: 10.2214/AJR.15.15962

40 Mango VL, Wynn RT, Feldman S, Friedlander L, Desperito E, Patel SN, Gomberawalla A and Ha R: Beyond wires and seeds: Reflector-guided breast lesion localization and excision. Radiology 284(2): 365-371, 2017. PMID: 28430555. DOI: 10.1148/radiol.2017161661

41 Falcon S, Weinfurtner RJ, Mooney B and Niell BL: SAVI SCOUT $^{\circledR}$ localization of breast lesions as a practical alternative to wires: Outcomes and suggestions for trouble-shooting. Clin Imaging 52: 280-286, 2018. PMID: 30193186. DOI: 10.1016/ j.clinimag.2018.07.008

42 Krischer B, Forte S, Niemann T, Kubik-Huch RA and Leo C: Feasibility of breast MRI after sentinel procedure for breast cancer with superparamagnetic tracers. Eur J Surg Oncol 44(1): 74-79, 2018. PMID: 29217399. DOI: 10.1016/j.ejso.2017.11.016

Received February 10, 2020

Revised February 24, 2020

Accepted February 27, 2020 